Proceeding Paper

\title{
Influence of Aging on the Structure and Magnetic Properties of Surface-Deposited Single-Molecule Magnets ${ }^{\dagger}$
}

\author{
Oleksandr Pastukh * and Łukasz Laskowski
}

Citation: Pastukh, O.; Laskowski, Ł. Influence of Aging on the Structure and Magnetic Properties of SurfaceDeposited Single-Molecule Magnets. Mater. Proc. 2021, 4, 81. https:// doi.org/10.3390/IOCN2020-07944

Academic Editors: Ana María Díez-Pascual, Antonio Di Bartolomeo and Guanying Chen

Published: 11 November 2020

Publisher's Note: MDPI stays neutral with regard to jurisdictional claims in published maps and institutional affiliations.

Copyright: (C) 2020 by the authors. Licensee MDPI, Basel, Switzerland. This article is an open access article distributed under the terms and conditions of the Creative Commons Attribution (CC BY) license (http://creativecommons.org/licenses/by/4.0/).

\author{
Institute of Nuclear Physics Polish Academy of Sciences, PL-31342 Krakow, Poland; \\ lukasz.laskowski@ifj.edu.pl \\ * Correspondence: oleksandr.pastukh@ifj.edu.pl \\ + Presented at the 2nd International Online-Conference on Nanomaterials, 15-30 November 2020; \\ Available online: https://iocn2020.sciforum.net/.
}

\begin{abstract}
In this paper, the main issues concerning the possibility of the organization of Mn12-based single-molecule magnets (SMMs) on the surface of silica nanostructures with the preservation of its structure and magnetic properties were summarized. The aging effects on structural and magnetic properties in Mn12-stearate SMMs deposited on the surface of spherical silica nanoparticles were also discussed.
\end{abstract}

Keywords: surface functionalization; single-molecule magnets; silica; surface deposition; magnetic aging

\section{Introduction}

Single-molecule magnets are a new class of nanoscale materials with unique magnetic properties of purely molecular origin [1]. Such structures possess high spin of the ground state and large magnetic anisotropy, which opens up great potential in terms of their applications in nanoelectronics [2]. The realization of high-performance nanoelectronic devices based on the manipulation of SMMs requires the surface deposition of such entities. The organization of individual bistable magnetic molecules on the surface, however, demands the precise chemical method and searching of proper supporting material, since any changes in structure may influence the magnetic properties [3].

Since for practical application it is important to follow the changes of SMMs magnetic performance over a long period of time, here, we aimed to discuss the structure modifications and their influence on the magnetic properties of such surface deposited entities. The discussion is based on the series of articles, dedicated to the synthesis, structural and magnetic properties investigation of Mn12-based SMMs deposited on the surface of silica nanostructures [4-7].

\section{Discussion}

The synthesis procedure of the investigated material was based on anchoring the individual SMMs onto the silica support using propyl carbonic acid groups [4]. As the substrate, spherical silica nanoparticles (of diameter about $300 \mathrm{~nm}$ ) were chosen. They possesses a large specific surface area and functional units that are placed at the external surface of this material, what is important for large molecules separation. The Mn12-stearate SMMs were used for surface deposition, as they have a total spin $S=10$ of the ground state and also possess high structural stability due to the outside bulky ligands, are soluble in most organic solvents and stable against water catalyzed reduction [8].

At first, to confirm successful deposition of the molecules on the surface, transmission electron microscopy (TEM) measurements were carried out. On the TEM images at high magnification, individual molecules of Mn12-stearate SMMs were unambiguously 
observed in the form of islands [4,5]. Then, the performed Raman spectroscopy analysis was applied to confirm correct anchoring of the molecules on the surface. The presence of all the typical bands, corresponding with the vibrations of stearate groups, were observed, which confirms the preservation of structure of SMMs after deposition [6]. What is most important is that the retention of the magnetic properties was confirmed with the use of SQUID magnetometry. The magnetic measurements show retention of magnetic hysteresis in $\mathrm{M}(\mathrm{H})$ dependence and the presence of a slow relaxation process in molecules after surface deposition [6].

In order to analyze how the characteristics of such materials change over a long time period, the aging effect was investigated [7]. The aging studies (over a period of about seven months) revealed a significant decrease in basic magnetic parameters (an exponential decrease of the coercive field and remnant magnetization) and change in magnetic relaxation behavior. The Raman spectroscopy measurements revealed gradual degradation of the compound and formation of the hydroxyl stearate system over time, which explains the observed magnetic changes. Additionally, the aged samples were compared with the as-synthesized material after thermal decomposition. For both samples, similar bands at the $\mathrm{MnO}$ region of Raman spectra as well as the partial covering of hysteresis loops were observed, which confirms that the structural modifications caused by the temperature, affect the material in a similar way as the aging process.

Author Contributions: Conceptualization, Ł.L. and O.P.; methodology, Ł.L. and O.P.; investigation, Ł.L. and O.P.; writing-original draft preparation, O.P.; writing-review and editing, Ł.L. All authors have read and agreed to the published version of the manuscript.

Funding: This research was funded by National Science Centre, grant number: 2017/26/E/ST5/00162.

Acknowledgments: The authors are grateful to Piotr Konieczny, Magdalena Laskowska and Dominik Czernia for fruitful discussions and support during the research.

Conflicts of Interest: The authors declare no conflict of interest.

\section{References}

1. Sessoli, R.; Gatteschi, D.; Caneschi, A.; Novak, M. Magnetic bistability in a metal-ion cluster. Nature 1993, 365, $141-143$.

2. Bartolomé, J.; Luis, F.; Fernández, J.F. Molecular Magnets: Physics and Applications; Springer: Berlin/Heidelberg, Germany, 2013; 395 .

3. Cornia, A.; Mannini, M.; Sainctavit, P.; Sessoli, R. Chemical strategies and characterization tools for the organization of single molecule magnets on surfaces. Chem. Soc. Rev. 2011, 40, 3076-3091.

4. Laskowski, L.; Kityk, I.; Konieczny, P.; Pastukh, O.; Schabikowski, M.; Laskowska, M. The Separation of the Mn12 Single-Molecule Magnets onto Spherical Silica Nanoparticles. Nanomaterials 2019, 9, 764.

5. Laskowska, M.; Pastukh, O.; Kuźma, D.; Laskowski, Ł. How to Control the Distribution of Anchored, Mn12-Stearate, SingleMolecule Magnets. Nanomaterials 2019, 9, 1730.

6. Laskowska, M.; Pastukh, O.; Konieczny, P.; Dulski, M.; Zalsiński, M.; Laskowski, L. Magnetic behaviour of Mn12-stearate singlemolecule magnets immobilized on the surface of $300 \mathrm{~nm}$ spherical silica nanoparticles. Materials 2020, $13,2624$.

7. Pastukh, O.; Konieczny, P.; Czernia, D.; Laskowska, M.; Dulski, M.; Laskowski, Ł. Aging effect on the magnetic properties of Mn12stearate single-molecule magnets anchored onto the surface of spherical silica nanoparticles. Mater. Sci. Eng. B 2020, $261,114670$.

8. Park, C.D.; Jeong, D.Y. Soluble Single-Molecule Magnet: Mn12-stearate. Bull. Korean Chem. Soc. 2001, 22, 611-615. 